\title{
Case Report: Pregnancy with Fetal Hydrathorax
}

\author{
Aditya Arya Putra1*, Rahmad Rizal Budi Wicaksono1, Besari Adi Pramono, Herman \\ Kristanto ${ }^{1}$, Arsita Ekarini ${ }^{2}$
}

\author{
1Department Obstetrics and Gynaecology, Faculty of Medicine, Diponegoro University, Kariadi General Hospital, \\ Indonesia \\ ${ }^{2}$ Department of Pediatrics, Faculty of Medicine, Diponegoro University, Kariadi General Hospital, Indonesia
}

Keywords:

Case report

Extrauterine intrapartum treatment (EXIT)

Fetal hydrothorax (FHT)

Thoracoamniotic shunt (TAS)

\footnotetext{
*) Correspondence to: dr.adityaaryaputra@yaho o.co.id
}

Article history:

Received 24-05-2021

Accepted 30 -06-2021

Availableonline10-12-2021

\begin{abstract}
Background: Cases of fetal hydrothorax (FHT) are rare, with an occurrence probability of 1 in every 10,000-15,000 pregnancies. The condition may remain undiagnosed, and the fetus may be aborted, or death may occur soon after birth in outlying hospitals before transfer to a tertiary care center. The incidence rate of FHT is higher in males than females $(2: 1)$. One of the most effective methods of diagnosing fetal hydrothorax is sonography. Three forms of currently available treatments are: thoracentesis, thoracoamniotic shunting (TAS), and thoracomaternal cutaneous drainage. Fetal outcomes could be improved by performing the Extrauterine Intrapartum Treatment (EXIT) procedure

Case Presentation: We present two cases of first pregnancy primary FHT. In the first case, a 24-year-old woman was diagnosed with asymptomatic FHT in the 28th week of gestation without any prior history. In the second case, a 22 -year-old woman with poor medical history was diagnosed in the 35th week of pregnancy and was experienced difficulty of breathing. Both pregnancies were delivered by cesarean section based on obstetric indications. Thoracentesis was performed on both neonates, and pathological examination of the pleural fluid was conducted. However, they died shortly after birth.

Conclusion: The EXIT procedure is still a challenging method. A fetus with FHT is at significant risk of pulmonary hypoplasia and respiratory distress following delivery. Early diagnosis and intervention of FHT are vital to ensure a good prognosis. Approaching multidisciplinary groups, providing supportive diagnostic facilities and financial support is essential in improving fetal outcomes and preventing FHT in subsequent pregnancies.
\end{abstract}

DIMJ, 2021, 2(2), 67-72 DOI: https:// doi.org/10.14710/dimj.v2i2.11060

\section{Introduction}

The term fetal hydrothorax describes fluid accumulation in the pleural cavity area, either unilaterally or bilaterally. ${ }^{1,23}$ Cases of fetal hydrothorax (FHT) are rare, with a probability of occurring in 1 out of every 10,000-15,000 pregnancies. ${ }^{13,15}$ The incidence of primary FHT may be even higher, considering that the condition may remain undiagnosed in many cases. Primary FHT may resolve spontaneously. The fetus may be aborted, or death may occur soon after birth in outlying hospitals before transfer to a tertiary care center. $^{2,3,6}$ The incidence rate for FHT is higher in males than females (with a ratio of 2:1). ${ }^{4,5}$ Cases of FHT occur due to primary or secondary chylous leaks from fluid retention related to immune or nonimmune hydrops. One of the most effective methods of diagnosing FHT is sonography. Although the earliest gestational age at which prenatal diagnosis has been made using sonography is 17 weeks, most cases are not detected until after 30 weeks. ${ }^{3}$ Upon discovery, cases of FHT can be managed conservatively or actively through the fetus. There are three forms of treatment available for managing fetuses with FHT: thoracentesis, thoracoamniotic shunting (TAS), and thoracomaternal cutaneous drainage. However, all three options do not provide a high fetal survival rate and likely interfere with the pregnancy condition. ${ }^{3,7,10}$ The Extrauterine Intrapartum Treatment (EXIT) provides alternative to improve the neonatal outcomes. In this report, we will present two cases, both of which are first-time pregnancies at 36 weeks of gestation that have been diagnosed with FHT. 


\section{Case Presentation}

\section{Case 1}

A 24-year-old married woman with G1P0A0 was in the 36th week of gestation. She lived in Semarang, Central Java, Indonesia. She was an inpatient at Dr. Kariadi Hospital in Semarang at the time of the study. There was no record of known illnesses in her medical history. However, there was no complete medical examination. The antenatal care was routinely performed without any complication. However, on her gestational age of 28 weeks, the fetus was diagnosed with FHT using ultrasound. Afterward, the fetal condition was observed every 12 weeks. Unfortunately, the patient discontinued her antenatal care until she felt the onset of contractions and admitted in the emergency department due to premature labor at 36 weeks of gestation. We performed an emergency caesarean section with informed consent from the patient. The caesarean section operation was conducted successfully; however, the baby did not cry immediately upon delivery. The Perinatology team immediately performed resuscitation.

The newborn's weight was 3200 grams with an APGAR score of one in the 1st, 5th, and 10th minute. After the resuscitation was completed, the Thoracic Vascular Surgery team performed thoracentesis. At the time of the procedure, $\pm 110 \mathrm{cc}$ of serosa fluid from the left side of the thorax and 40 cc haemorrhagic serosa fluid from the right side of the thorax was removed. Further anatomical pathology examinations identified the fluid as lymphocytic effusion. After receiving treatment, the baby was placed into the neonatal intensive care unit (NICU) and died 30 minutes later.

\section{Case 2}

A 22-year-old married woman with G1P0A0 was in the 36th week of gestation and lived in Jepara, Central Java, Indonesia. This patient was referred to RSUP Dr. Kariadi due to polyhydramnios at 35 weeks of gestation accompanied with difficulty of breathing. On her first visit to our center, her pregnancy was diagnosed with FHT. The patient was then followed up by a multidisciplinary team consisting of Obstetrics and Gynaecology, Perinatology, and Vascular Thoracic Surgery. The patient was planned for an elective caesarean section with EXIT procedure at the 38th week of gestation. Corticosteroid course (dexamethasone $6 \mathrm{mg} / 12 \mathrm{~h}$ for two days) to accelerate the fetal lung maturation was given. However, due to the ruptured of the membrane, patient entering the state of labor, the team eventually decided to conduct an emergency caesarean section in the 36 th week of gestation. The caesarean section operation was successful, and the baby began to cry weakly upon delivery. The perinatology team immediately performed resuscitation.

The newborn's weight was 2750 grams with an APGAR score of seven at the 1st minute, eight at the 5th minute, and nine at the 10th minute. Once the Perinatology team had completed the resuscitation, the Thoracic Vascular Surgery team performed an ultrasound-guided thoracentesis on the baby's left thorax. At the time of the procedure, the fluid consisted of $40 \mathrm{cc}$ serous fluid, and daily fluid aspiration evaluations in the NICU unit were scheduled with a maximum of $50 \mathrm{cc}$ aspiration. The results from anatomical pathology examinations identified the fluid caused by nonspecific inflammation and did not discover any malignancies. After four days of treatment, saturation levels in the newborn were only around $80 \%$. Unfortunately, the baby was declared dead the next day.

\section{Discussion}

The two cases were different from the point of view of diagnosis, symptoms in pregnancy, and the antepartum interventions. The first case was diagnosed in the 28th week of gestation. The evaluation of the fetus then ensued after considering several factors, such as the absence of hydramnios. We expected a spontaneous resolution of the fluid in the fetal thorax cavity; however, there was never any sign of a decrease in fluid level. The second case was diagnosed near the early term even after a possible congenital fetal condition was suggested in the secondary health facility due to the detection of polyhydramnios. The symptom of shortness of breath that the second patient reported could be accounted for by the presence of these polyhydramnios, something that she had thought to be expected in pregnancy.

In addition to the diagnosis, the interventions of both cases were also distinct. The first patient was not given corticosteroid to help fetal lung maturation due to negligence of routine antenatal care after the diagnosis of FHT was made. The second patient was given a corticosteroid course of dexamethasone $6 \mathrm{mg}$ every 12 hours for two days intramuscularly. Even though antenatal interventions were not conducted, thoracentesis was performed promptly after the fetuses were born in an attempt to improve prognosis. Although both newborns eventually died, the distinction was seen in the survival duration. Unlike the first newborn who died immediately after birth, the second newborn managed to survive for four days in the NICU, where he eventually died due to many 
complications of FHT. An extrauterine intrapartum treatment (EXIT) procedure had been discussed as a possibility for both babies. EXIT is a management strategy that allows interventions on a neonate delivered from the uterus with fetoplacental circulation preserved and it is a novel technique which provides a therapeutic option for fetuses with a variety of potentially fatal anomalies, particularly airway anomalies. ${ }^{12}$ However, the attempt of EXIT procedure was failed to prolong the survival of the baby in the second case. After we conducted discussion and examinations to determine the primary cause of death, it was concluded that both cases were different. The first newborn died from respiratory disorder (Persistent Pulmonary Hypertension of the Newborn (PPHN)), and the second baby succumbed to the complication of Total Anomalous Pulmonary Venous Drainage (TAPVD) that also caused a respiratory disorder (PPHN).

The natural history of FHT is significantly different from that of chylothorax in newborns and carries a much poorer prognosis. The mortality rate for chylothorax in newborns is $15 \%$ at most, while the mortality rate for prenatally diagnosed FHT is $53 \%$. $^{2,5,16}$ However, this should also be separated from the finding that several characteristics of primary FHT are associated with a more favorable outcome. For example, unilateral FHT without evidence of tension such as mediastinal shift or diaphragmatic eversion is associated with $100 \%$ survival. ${ }^{2,3}$ However, this differed with the survival rate of merely $52 \%$ in bilateral FHT. ${ }^{2}$ Survival was also observed in all cases of FHT that spontaneously resolve, which occurred in approximately $5 \%$ to $22 \%$ of cases. ${ }^{2,14,17-22}$ Nonetheless, due to the possibility of a positive-spontaneous regression, a period of observation is required in each case of FHT. ${ }^{7}$

Early diagnosis in fetal hydrothorax might help the management of pregnancy and also the fetus. Several measures can be taken once we successfully diagnose FHT. Prenatal karyotyping is recommended, especially if fetal intervention is considered. The incidence of Down syndrome in FHT is $4.9 \% .{ }^{6}$ Since the incidence of associated congenital heart disease may be as high as 5\%; we recommend that every fetus diagnosed with FHT undergoes fetal echocardiography. ${ }^{23}$ We also recommend that these pregnancies be followed closely, with ultrasound examinations every 1 to 2 weeks for early detection of signs consistent with tension hydrothorax such as mediastinal shift, diaphragmatic eversion, development of hydrops, and polyhydramnios. ${ }^{7}$

The management of pleural effusion in a fetus is highly complicated, with the difficulty of distinguishing the fetal pleural effusion between primary and secondary FHT. Secondary FHT is much more common in fetuses than in infants. ${ }^{1,3}$ Secondary FHT may be caused by a wide variety of maternal and fetal disorders, including chromosomal anomalies; cardiovascular, hematologic, gastrointestinal, pulmonary, metabolic, infectious, and neoplastic disorders; and malformations of the placenta and the umbilical cord..$^{3,4,9-11}$ Such cases are also a challenge for obstetricians and gynaecologists to diagnose. Theoretically, the diagnosis can be made in the 30th week of gestation. ${ }^{3}$ To distinguish the leading cause of whether FHT is primary or secondary, a study demonstrated that $40 \%$ of secondary FHT cases had major congenital abnormalities in the fetus, which were detected using sonographic examinations., ${ }^{3,22}$ Some cases still required fetal thoracentesis to distinguish primary from secondary FHT. ${ }^{5}$ However, no underlying causes were found in most cases of fetal hydrothorax. ${ }^{7}$

If the diagnosis is late or there is no intervention in early pregnancy after a diagnosis of FHT, fetuses will suffer a significant risk of pulmonary hypoplasia and respiratory distress following delivery. We recommend that fetuses with a large pleural effusion be transferred to and delivered within a tertiary care center. Prenatal consultations with a pediatric surgeon, neonatologist, geneticist, and pediatric cardiologist are also recommended. The presence of FHT does not influence the mode of delivery, and cesarean delivery should be performed based on obstetric indications.

Several fetal management options can be taken when pregnancy with FHT is diagnosed. During pregnancy, a thoracoamniotic shunt (TAS) can be inserted. However, in Indonesia, this procedure can only be carried out at the Harapan Kita Mother and Child Hospital in Jakarta. Once the baby is born, the options are to do either extrauterine intrapartum treatment (EXIT) or thoracentesis. The EXIT procedure is performed when the baby is born before cutting the umbilical cord, while thoracentesis is done after cutting the umbilical cord, with the EXIT survival rate reaching up to $100 \%$ in some case series. ${ }^{24}$ In a case report of non-hydrops cases, the survival rate was reported to be high. However, there was no significant difference between taking any action versus taking no action $(98.1 \%$ survival rate without fetal therapy, $96.3 \%$ with thoracentesis, $100 \%$ with TAS). The difference in survival rates between EXIT and thoracentesis procedures has never been mentioned in previous literature. Some neonatologists believe that prenatal decompression is closely related to the rate of resuscitation required in newborns. ${ }^{9,26}$ There are no data to support this claim, 
although it was suggested that delaying thoracentesis until delivery reduces fetal and maternal risk.

According to previous studies, out of 287 cases of primary FHT, the survival rate for cases with hydrops was $58.0 \%(113 / 195)$ versus non-hydrops at $97.8 \%$ (90/92). The survival rate in hydropic cases for the group of patients without therapy was $59.7 \%$ (40/67), 51.5\% (35/68) for those with thoracentesis, and $63.3 \%(38 / 60)$ in those who received thoracoamniotic shunting (TAS), while in nonhydropic cases the survival rates were $98.1 \%$ (53/54) for those who received no therapy, $96.3 \%$ (26/27) for thoracentesis, and $100 \%$ (11/11) with TAS. Therefore, the most highly recommended therapy in primary FHT is thoracoamniotic shunting (TAS) as a procedure with the highest survival rate. ${ }^{25}$

There are several take-home points from the two cases that have been presented. Early diagnosis followed by early interventions may increase the prognosis of fetal outcomes. The attempt to evaluate the fetal hydrothorax with imaging proved challenging, especially regarding ultrasound. In theory, FHT may be diagnosed as early as the $17^{\text {th }}$ week of pregnancy. Nonetheless, in these two cases, both FHT was diagnosed in the $3^{\text {rd }}$ trimester. The management was further complicated by the regulation required to perform EXIT. Prior simulations and training are also compulsory for the health workers involved in the procedure. The application of shunt in pregnancy with FHT as a compounding complication is still difficult even for OB/GYN colleagues, whereas it is necessary to ameliorate the fetal prognosis. The patients also experienced predicaments in transferring to tertiary health facilities due to the location of residence and the lack of transportation methods. This problem was particularly true for the second patient, in which the city she resides is different from her primary care facility is situated. Furthermore, the tertiary care center location, RSUP Dr. Kariadi, is located in a different city from both her residence and primary care. At the end of care, both patients were content with the pregnancy and felt relieved from delivery management, allowing them to go back to daily activities soon. They hoped that health workers could help them plan their subsequent pregnancies to reduce the risk of occurrence equal to this pregnancy and have healthy newborns in the future.

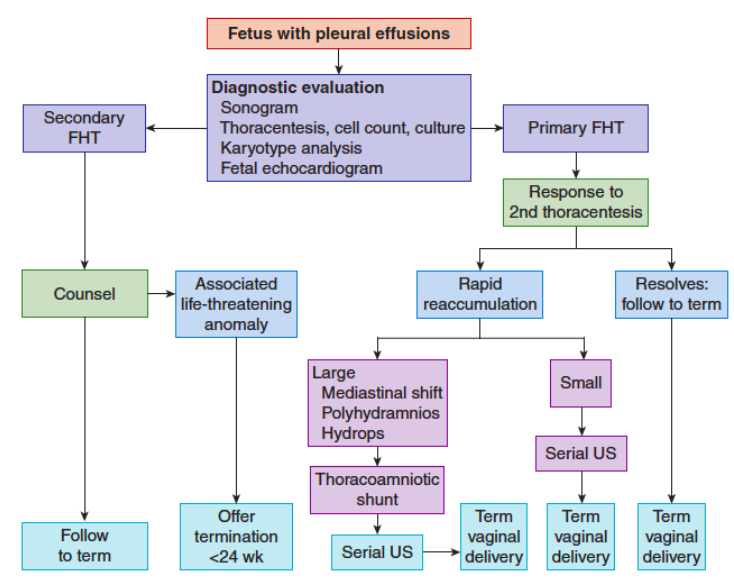

Figure 1.Algorithm for the management of fetal pleural effusions. (taken from Thoracoamniotic Shunting for Fetal Hydrothorax: Predictors of Intrauterine Course and Postnatal Outcome. $)^{7}$

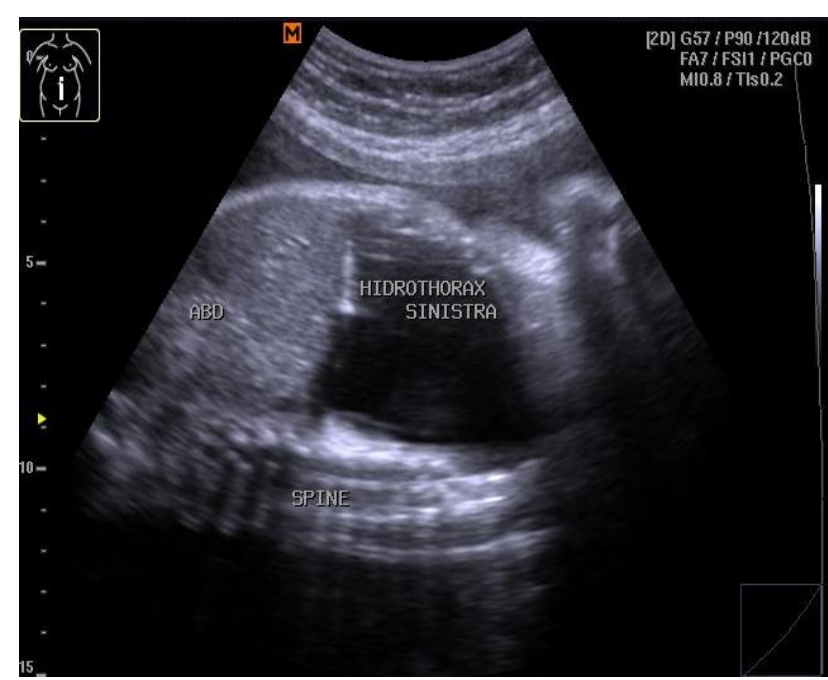

Figure 2.Diagnostic ultrasound at 28th weeks of gestation (Case 1)

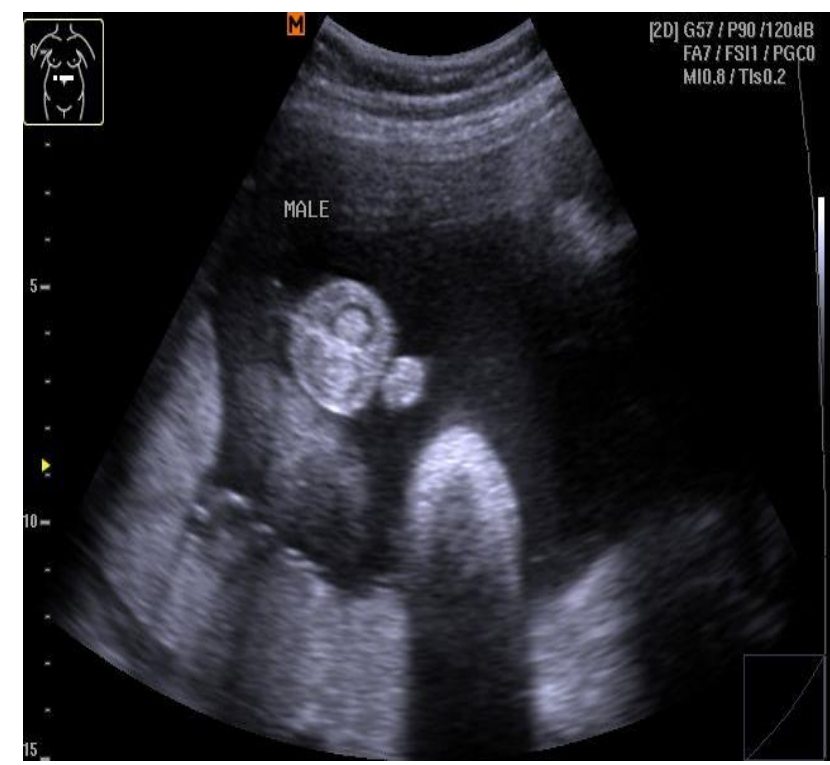

Figure 3. Ratio fetal hydrothorax male to female 2:1 (Case 1) 


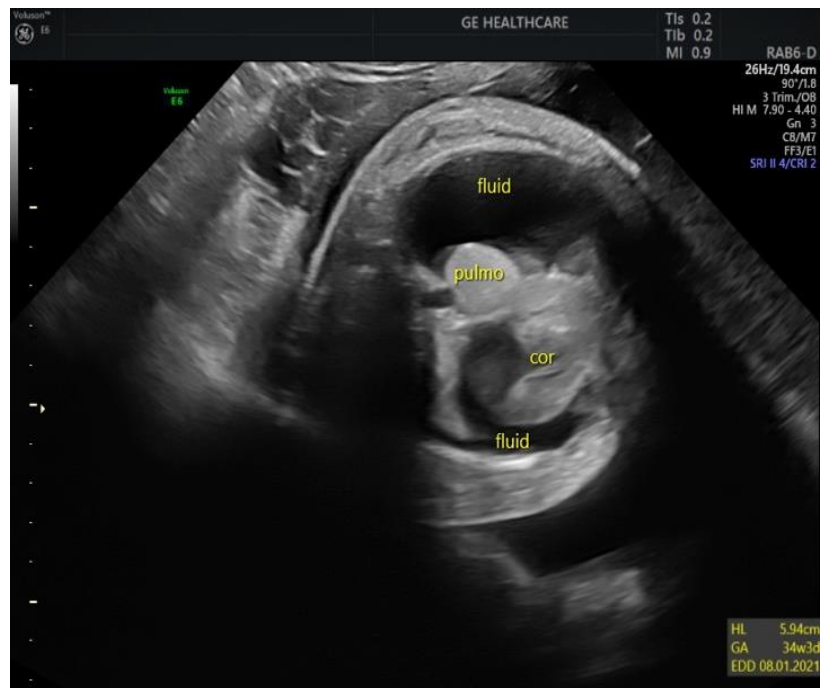

Figure 4.Ratio fetal hydrothorax male to female 2:1 (Case 1)

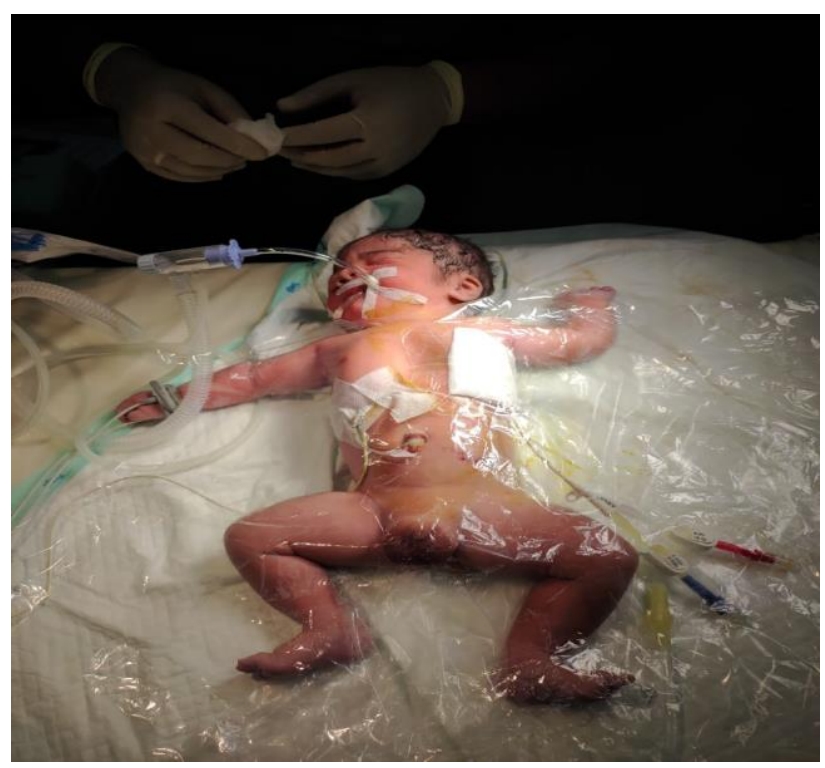

Figure 5. Fetal thoracocentesis

\section{Conclusion}

Cases of Fetal Hydrothorax are sporadic and closely related to fetal outcomes. Pregnancy itself can be highly complicated if there is abortion, polyhydramnios, fetal death in the womb, preterm labor, or congenital abnormalities occurring in the fetus such as Down syndrome or congenital heart abnormalities. However, the possibility of spontaneous resolution should be considered as a significant factor in some cases. In this study, FHT was more commonly found in male fetuses. Both cases also experienced preterm labor. Bilateral FHT could only be identified after birth due to the difficulty of intrapartum detection with imaging. This delay in diagnosis resulted in poor newborn outcomes despite the subsequent high-level management given in tertiary healthcare facilities. Consequently, newborns from the first and second cases died within 30 minutes and four days after birth, respectively. In the future, early diagnosis and intervention of FHT are vital to ensure a good prognosis. Approaching multidisciplinary groups and providing supportive diagnostic facilities, sources of financial support, and antenatal care discipline are essential in optimizing fetal outcomes and preventing pregnancy with FHT in subsequent pregnancies.

\section{Conflicts of Interest}

The authors declare no conflict of interest.

\section{Funding}

No specific funding was provided for this article.

\section{Acknowledgments}

This work was supported by Department of Obstetrics and Gynaecology, and Department of Pediatrics, Faculty of Medicine, Diponegoro University.

\section{References}

1. O'Brien B, Kesby G, Ogle R, Rieger I, Hyett JA. Treatment of primary fetal hydrothorax with OK-432 (Picibanil): Outcome in 14 fetuses and a review of the literature. Fetal Diagn Ther. 2015;37(4):259-66.

2. Longaker MT, Laberge JM, Dansereau J, et al. Primary fetal hydrothorax: natural history and management. J Pediatric Surg. 1989;24:573576.

3. Biachi DW, Crombleholme TM, D'Alton ME, Malone FD. Fetology. $2^{\text {nd }}$ Ed. United States: Mc.Graw-Hill. 2010:255-262.

4. Hurton Toni, BS R, Morril Heather R, Bromley Bryann M. Fetal Hydrothorax a Potentially Dynamic and Variable Condition. Case Rep. 1997;JDMS 12:132-135

5. Cao L, Du Y, Wang L. Fetal pleural effusion and Down syndrome. Intractable Rare Dis Res. 2017;6(3):158-62.

6. Petersen S, Kaur R, Thomas JT, Cincotta R, Gardener G. The outcome of isolated primary fetal hydrothorax: A 10-year review from a 
tertiary center. Fetal Diagn Ther. 2013;34(2):69-76.

7. Mallmann MR, Graham V, Rösing B, Gottschalk I, Müller A, Gembruch U, et al. Thoracoamniotic Shunting for Fetal Hydrothorax: Predictors of Intrauterine Course and Postnatal Outcome. Fetal Diagn Ther. 2017;41(1):58-65.

8. Hache JJ, Emery SP, Vallejo MC. Bilateral fetal hydrothorax requiring intrauterine fetal thoracoamniotic shunts: Anesthetic considerations and management. ScientificWorldJournal. 2009;9:435-40.

9. Prontera W, Jaeggi ET, Pfizenmaier M, Tassaux D, Pfister RE. Ex utero intrapartum treatment (EXIT) of severe fetal hydrothorax. Arch Dis Child Fetal Neonatal Ed. 2002;86(1):58-61.

10. Rustico MA, Lanna M, Coviello D, Smoleniec J, Nicolini U. Fetal pleural effusion. Prenat Diagn. 2007;27(9):793-9.

11. Hidaka Nobuhiro MD, Yoshihide Chiba, M.D. PD. Fetal Hydrothorax Resolving Completely After a Single Thoracentesis. J Reprod Med. 2007;52

12. Hidaka Nobuhiro MD, Yoshihide Chiba, M.D. PD. Fetal Hydrothorax Resolving Completely After a Single Thoracentesis. J Reprod Med. 2007;52.

13. Henry PY, Aravindan CS, Sivakumar K, Krishna HR. Extrauterine Intrapartum Treatment (EXIT) in bilateral primary fetal hydrothorax. Indian J Pediatr. 2009;76(1):99101.

14. Bianchi S, Lista G, Castoldi F, Rustico M. Congenital primary hydrothorax: Effect of thoracoamniotic shunting on neonatal clinical outcome. J Matern Neonatal Med. 2010;23(10):1225-9.

15. Lim-navarro BL, Gorgonio NM. A dilemma in the management of fetal pleural effusion: A case report of two cases. Dep Obstet Gynecol Cardinal Santos Med Cent. 2017;

16. Khalil BA, Jesudason EC, Featherstone NC, Sarginson R, Kerr S, Ashworth M, et al. Hidden pathologies associated with (and concealed by) early gestational isolated fetal hydrothorax. J Pediatr Surg. 2005;40(7):7-9.

17. Derderian SC, Trivedi S, Farrell J, Keller RL, Rand L, Goldstein R, et al. Outcomes of fetal intervention for primary hydrothorax. J Pediatr Surg [Internet]. 2014;49(6):900-4.

18. Mon RA, Treadwell MC, Berman DR, Day L, Kreutzman J, Mychaliska GB, et al. Outcomes of fetuses with primary hydrothorax that undergo prenatal intervention (prenatal intervention for hydrothorax). J Surg Res [Internet]. 2018;221(734):121-7.

19. Haskell, C. M., G. P. Canellos, B. G. Leventhal, P. P. Carbone, J. B. Block, A. A. Serpick and OSS. Long-Term In Utero Drainage Of Fetal Hydrothorax. N Engl J Med. 2015;281(19):1028-34.

20. Trocado V, Coutinho-Borges JP, Carlos-Alves M, Santos J, Pinheiro P. Fetal primary hydrothorax with spontaneous resolution. Case Reports Women's Heal [Internet]. 2017;15(June):6-7.

21. Lam H, Yates R, Jauniaux E. Successful early in utero management of fetal hydrothorax in a twin pregnancy. Prenat Diagn. 2003;23(3):221-4.

22. Bagur Krishnamurthy M, Malhotra A. Congenital chylothorax: current perspectives and trends. Res Reports Neonatol. 2017; Volume 7:53-63.

23. Seiji Wada. The prognostic factors and outcomes of primary fetal hydrothorax with the effects of fetal intervention. Cent Matern Neonatal Reprod Med Natl Cent Child Heal Dev Tokyo, Japan. 2012.

24. Abraham RJ, Sau A, Maxwell D. A review of the EXIT (Ex utero Intrapartum Treatment) procedure. Journal of Obstetrics and Gynaecology. 2010;30(1):1-5. 10.3109/01443610903281656

25. Bigras JL, Ryan G, Suda K, Silva AE, Seaward PGR, Windrim R, et al. Echocardiographic evaluation of fetal hydrothorax: The effusion ratio as a diagnostic tool. Ultrasound Obstet Gynecol. 2003;21(1):37-40. 